\title{
Exploratory Research on Bearing Characteristics of Confined Stabilized Soil
}

\author{
Shuai Shuai WU, Zheng Guo GAO, Shi Yang LI, Wen Bo CUI, Xin HUANG \\ School of Transportation Science and Engineering, Beihang University, Beijing 100191, China
}

\begin{abstract}
The performance of a new kind of confined stabilized soil (CSS) was investigated which was constructed by filling the stabilized soil, which was made by mixing soil with a binder containing a high content of expansive component, into an engineering plastic pipe. Cube compressive strength of the stabilized soil formed with constraint and axial compression performance of stabilized soil cylinders confined with the constraint pipe were measured. The results indicated that combining the constraint pipe and the binder containing expansion component could achieve such effects: higher production of expansive hydrates could be adopted so as to fill more voids in the stabilized soil and improve its strength; at the same time compressive prestress built on the core stabilized soil, combined of which hoop constraint provided effective radial compressive force on the core stabilized soil. These effects made the CSS acquire plastic failure mode and more than twice bearing capacity of ordinary stabilized soil with the same binder content.
\end{abstract}

\section{Introduction}

A confined stabilized soil pile (CSSP) is proposed in this paper as a new kind of foundation treatment method. It is constructed by filling the stabilized soil, which is made by mixing soil with a binder containing a high content of expansive component, into a constraint pipe with enough tensile modulus and strength fixed in ground.

It is supposed that combining the constraint of the pipe with the binder containing expansion component would bring several unique effects. 1) Volume expansion produced by expansive component in the binder can reduce voids in the stabilized soil so as to significantly enhance the strength of stabilized soil [1] [2]. Under the constraint conditions, higher content of the expansive component could be adopted so as to further improve strength of the stabilized soil. 2) Hoop constraint of confined concrete can enhance the bearing capacity (BC) obviously [3]; similar to this, hoop constraint of CSS should also enhance the BC greatly. 3) Bringing prestress on confined concrete can enhance its $\mathrm{BC}$ further [4]; according to this principle, under the constraint, the expansion produced by the binder might build prestress upon the core stabilized soil, which would enhance the radial confining force on the core stabilized soil and further enhance the $\mathrm{BC}$ when bearing load.

Combining these benefits together is expected to provide the CSSP with rather high $\mathrm{BC}$ and make it become an effective foundation treatment method. But whether this idea is feasible or not should be verified. No literature about research on the CSS has been found by the authors. Although the fact that $\mathrm{BC}$ of confined concrete is much higher than that of unconfined concrete have been proved,

*Corresponding author: Email: hx2000@buaa.edu.cn whether the result fit the CSS or not is unconfirmed. Therefore the performance of the CSS was investigated in this exploratory research.

\section{Experiment Schemes and Methods}

\subsection{Experiment Scheme}

1) Experiment one: cube compressive strength of the stabilized soil. The binder content of 15 and the mass ratio of cementing component and expansive component $(\mathrm{C} / \mathrm{E})$ of $7 / 3,6 / 4$ and $5 / 5$ were chosen in this experiment. For each $\mathrm{C} / \mathrm{E}$, stabilized soil samples formed with constraint (experimental group) and those formed without constraint (control group) were prepared; in addition, an expansion group was also included in which five faces of the cubic stabilized soil samples were confined and one face was left free for measuring the expansion quantity during curing time.

2) Experiment two: axial compression performance of the confined stabilized soil cylinder(CSSC). The effects of confinement stiffness (CS, product of tensile modulus and thickness of the constraint pipe) and density of the stabilized soil on bearing behavior of the CSSC were considered. $\mathrm{C} / \mathrm{E}=6 / 4$ and binder content $=15 \%$ were chosen, with which the stabilized soil samples formed with constraint reached the highest strength according to the results of experiment one. Polyvinyl chloride (PVC) pipe was adopted as the constraint pipe, whose CS was adjusted by changing its wall thickness. The density of the stabilized soil was adjusted by changing water content of the soil; a plastic state soil with optimum water content and a plastic-flow state soil with water content higher than 
liquid limit were chosen respectively. Two groups of stabilized soil cylinders were prepared, and both groups were formed with the constraint pipes, but the group of
CSSC bore load with the constraint pipe while the group of unconfined ones bore load without the constraint pipe. The scheme of Experiment two is shown in Table 1.

Table 1 Scheme of Experiment Two

\begin{tabular}{ccc}
\hline Number & Water content $(\%)$ & PVC pipe wall thickness $(\mathrm{mm})$ \\
\hline $\mathrm{C} 13-1 / 2 / 3$ & 13 & $2.2 / 2.5 / 3.2$ \\
$\mathrm{C} 30-1 / 2 / 3$ & 30 & $2.2 / 2.5 / 3.2$ \\
\hline
\end{tabular}

Physical indexes of the soil were shown in Table 2.

\subsection{Experimental Materials}

Table 2 Major Physical Indexes of the Experimental Soil

\begin{tabular}{ccccc}
\hline $\begin{array}{c}\text { Natural water content } \\
(\omega / \%)\end{array}$ & $\begin{array}{c}\text { Specific gravity of } \\
\text { soil particle }(\mathrm{Gs})\end{array}$ & $\begin{array}{c}\text { Natural } \\
\text { porosity }(\mathrm{e})\end{array}$ & $\begin{array}{c}\text { Liquid limit } \\
(\omega \mathrm{L} / \%)\end{array}$ & $\begin{array}{c}\text { Plastic limit } \\
(\omega \mathrm{P} / \%)\end{array}$ \\
\hline 20.4 & 2.61 & 0.66 & 28.9 & 12.7 \\
\hline
\end{tabular}

The cementing component of the binder, which would mainly produce calcium silicate hydrate $(\mathrm{CSH})$, consisted of Portland cement, slag, fluidized-bed combustion fly ash and hydrated lime, and the expansive component that would mainly produce calcium sulfoaluminate hydrate (AFt) was made of aluminate cement, gypsum and hydrated lime.

Elastic modulus and strength of the PVC pipe was 2753 and 31.89MPa respectively. The external diameter of the pipe was 11 centimeters, and its length was 20 centimeters. In order to eliminate vertical bearing function of the pipe, it was divided into 3 equal sections, and between each section there was an about $2 \mathrm{~mm}$-wide seam.

\subsection{Experiment Methods}

The method for preparing stabilized soil (according to JGJT 233) was generally as follows. Manually mix all kinds of components of the binder with water, and the water binder ratio was 0.5 . Then mix the mixture and soil sample by Type NJ-160 cement paste mixer. Slowly stir (62 r/min) for 1 minute and then quickly stir $(125 \mathrm{r} / \mathrm{min})$ twice for 2 minutes sequentially.

For the cubic samples, divide the mixture into three parts then push and hammer them in turn into a steel mold $(5 \mathrm{~cm} * 5 \mathrm{~cm} * 5 \mathrm{~cm})$, after that compact the mixture on the vibrating table for 2 minutes. As for preparation of the cylinders, divide the mixture into ten parts and push and hammer each part in turn into the constraint pipe. Finally cover the exposed face of the samples with plastic sheeting.

For the experimental group in Experiment one, the cubic stabilized soil samples were cured in room with average temperature of $20^{\circ} \mathrm{C}$ and average humidity of $42 \%$ during the first 14 days. Enough weights were pressed on the top of the steel mold to prevent expansion of the samples. During this period, cover them with cloth. At the 15 th day, unwrap the molds and put the samples into the standard curing box with temperature of $20 \pm 2^{\circ} \mathrm{C}$ and humidity of $95 \pm 2 \%$ for another 14 days. Cubic samples of the control group and the expansion group were cured in the same condition with those of the experimental group, while they were unmolded at the second day and the 15 th day respectively.

Vertically placed in water, the stabilized soil cylinders were cured in the room for 28 days, meanwhile press weights on the top of the cylinders.

In order to measure the expansion quantity, a dial gauge was placed vertically on the free face of the cubic stabilized soil samples during the first 14 days of the curing period. The strength of cubic samples were measured by pressed at the speed of $2.4 \mathrm{~mm} / \mathrm{min}$ with the Pavement Material Strength Experimenter $(0 \sim 30 \mathrm{KN})$. The cylinders were pressed at the speed of $0.1 \mathrm{kN} / \mathrm{s}$ by the microcomputer controlled electro hydraulic servo universal testing machine. Damage load, loaddisplacement curves and the failure modes were recorded.

\section{Results and Analysis}

The strength and the expansion data of experiment one were shown respectively in Figure 1. BC (damage load) and load-displacement curves of the cylinders were shown in Figure 2. And failure modes of some cylinders were shown in Figure 3. 


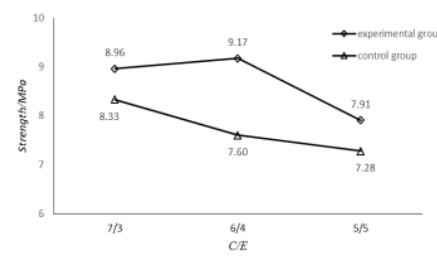

(a)

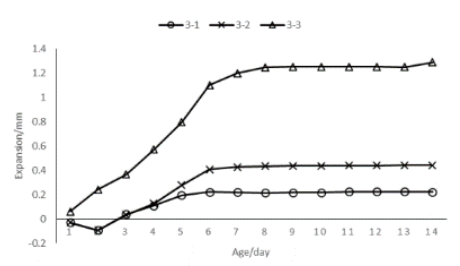

(b)

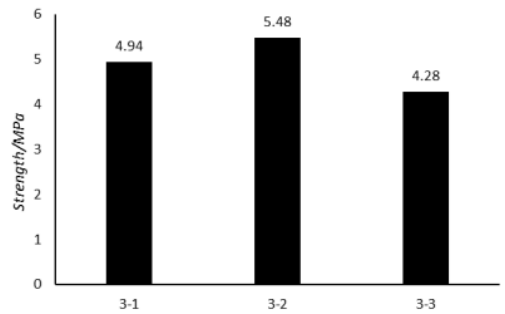

(c)

Figure 1 The Cubic Strength and Expansion Data of Experiment One

C13-2

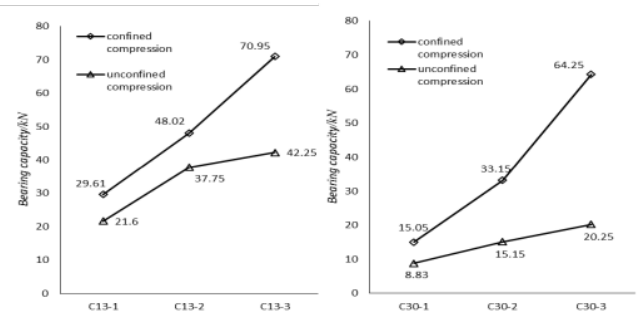

(a)

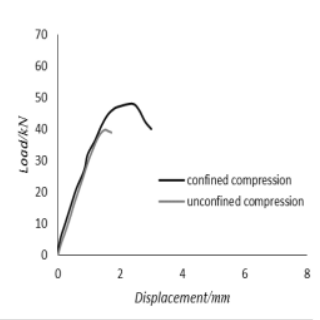

(b)

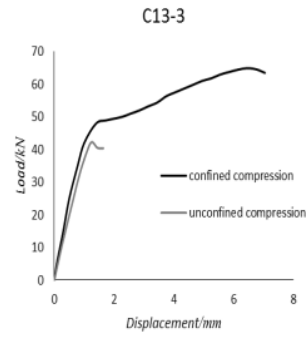

Figure 2 BC and Load-displacement Curves of Cylinders

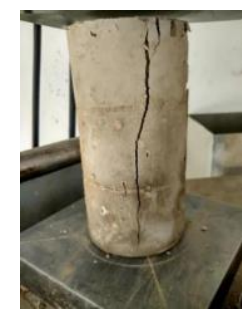

(a)

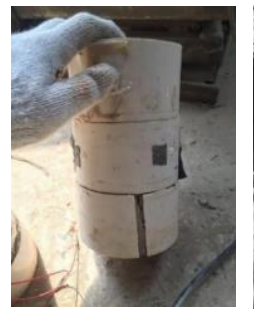

(b)

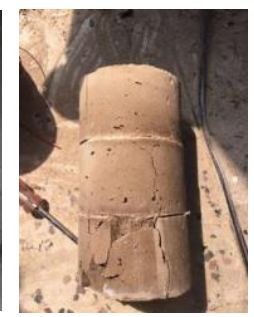

(c)

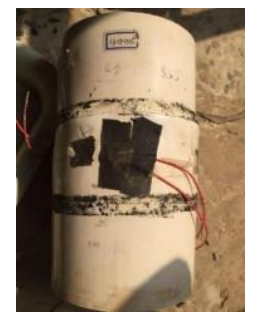

(d)

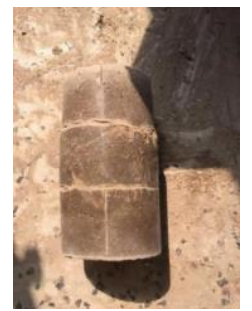

(e)

Figure 3 Failure Modes of Some Cylinders

\subsection{Compressive Strength of the Stabilized Soil Formed with Constraint}

Figure 1(a) showed that: strength of the stabilized soil formed without constraint reached its highest at $\mathrm{C} / \mathrm{E}=7 / 3$, and declined with $\mathrm{C} / \mathrm{E}$ reducing. While, as $\mathrm{C} / \mathrm{E}$ dropped strength of the stabilized soil formed with constraint increased at first then decreased, and reached the highest strength $9.17 \mathrm{MPa}$ at $\mathrm{C} / \mathrm{E}=6 / 4$, which was higher than the highest strength of the stabilized soil formed without constraint, 8.33MPa. Figure 1(b) showed that: apparent volume expansion emerged during the stabilized soil structure forming process in consequence of $\mathrm{AFt}$ developed. The expansion increased with the decrease of $\mathrm{C} / \mathrm{E}$. The strength of the corresponding stabilized soil, as shown in Figure 1(c), also increased first then decreased.

AFt generated volume expansion during the stabilized soil structure forming process. When the stabilized soil formed with constraint, instead of expanding outwards, AFt volume expansion would squeeze the internal space of the stabilized soil so as to decrease its porosity and increase the strength within a certain range of $\mathrm{C} / \mathrm{E}$. But it might also damage the stabilized soil structure formed by the cementation of $\mathrm{CSH}$, which led to the decrease of strength of the stabilized soil. The decrease of $\mathrm{C} / \mathrm{E}$ would lead the quantity of $\mathrm{CSH}$ to decrease but that of AFt to increase. When $\mathrm{C} / \mathrm{E}$ was less than a certain value, the quantity of $\mathrm{CSH}$ was too less to form sufficient cementation and too much expansion increased damage on the stabilized soil structure, which led to the decrease of strength of the stabilized soil. So the strength of sample 13 with $\mathrm{C} / \mathrm{E}=5 / 5$ greatly decreased.

As shown in Figure 2(a), BC of the unconfined stabilized soil cylinders (USSC) were related to the CS of the constraint pipe. Within a certain range of the CS, BC of the cylinders increased along with the increase of the CS.

If equal amount of the same binder was used, quantity of AFt volume expansion would be equivalent, and the expansion force put on the constraint pipe should be the same too. Hoop tensile deformation of the constraint pipe would generate under the expansion force. When the CS was small, a large proportion of AFt volume expansion expended outwards while only a little part of it left to squeeze the internal space of the stabilized soil. On this condition, the compactness and strength of the stabilized soil increased just in a limited range, such as C13-1 and C30-1 shown in Figure 2(a). With the increase of CS, the hoop tensile deformation of the constraint pipe decreased and volume of the cylinders grew a little under the same expansion effect. So a large part of the volume expansion contributed to squeeze the pores in the stabilized soil. Therefore, the compactness and strength of the stabilized soil increased significantly. These results indicated that in order to improve strength of stabilized soil CS should be 
high enough.

\subsection{Axial Compression Performance of CSSC}

As shown in Figure 2, BC of the CSSC was apparently higher than that of the unconfined ones, and about once higher could achieved as shown in C13-3 and C30-3 when strength of the stabilized soil of each pair of the cylinders were roughly the same. The load-displacement curves of the USSC were almost linear increasing before reaching the peak load then abruptly dropping, which presented a brittle failure mode. While the load-displacement curves of the CSSC could be roughly divided into two stages, the first was a linear stage, after which the deformation increased rapidly with load increasing until the curves reached the peak load and presented a plastic failure mode.

In the first stage the core stabilized soils were intact cemented bodies. The linear relationship between load and deformation of the cylinders, which were similar to that of unconfined ones, indicated that there were no apparent changes of properties of the stabilized soil. It could be deduced that in the stage the load was born by the core stabilized soils which kept intact state. As the load increased, radial strain of the core stabilized soil gradually reached its limit tensile strain and the core stabilized soil began cracking. In the second stage, as its integrality lost gradually, the core stabilized soil expanded horizontally and was compacted under the load, as a consequence of which considerable compressive deformation was presented and the constraint pipe bore increasing tension. It was the confining force of the constraint pipe that made the CSS bear load further. With the increase of the vertical load, tension stress in the constraint pipe increased constantly, until the pipe was broken as shown in Figure 3(b), or yielded as shown in Figure 3(d). Finally the CSSC lost bearing ability due to failure of the constraint pipe, and $\mathrm{BC}$ of the CSSC was determined by strength of the constraint.

\subsection{Compressive Prestress Building on the Confined Stabilized Soil}

AFt volume expansion under the constraint could be described in two stages as shown in Figure 6. Assuming there was no lateral constraint on the stabilized soil, during its structure forming expansion stress $\sigma_{1}$ made the diameter of stabilized soil samples expand freely from $\mathrm{L}_{0}$ to $L_{1}$; then a radial pressure $\sigma_{2}$ was applied to compress the samples to the final diameter $L_{2}$. Fig. 6 implied that if a CSS sample was prepared by using a binder containing expansive components, considerable confining force would be applied to the core stabilized soil during its structure forming, which indicated that there existed already radial compressive prestress on the stabilized soil before it bore vertical load.

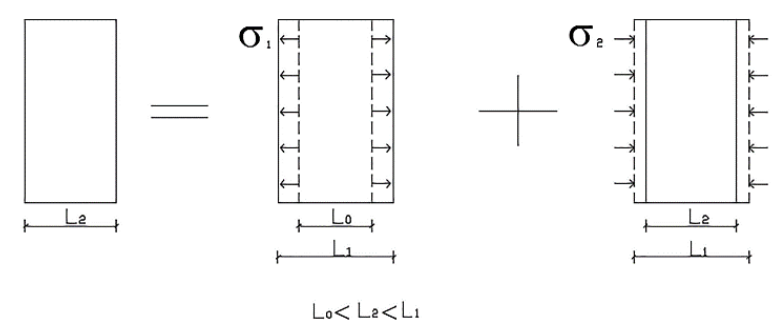

Figure 4 Section Sketch Drawing of Prestress Generation

The radial compressive prestress could enhance the radial confinement effect of the constraint pipe on the core stabilized soil when it bore vertical load, so as to further increase its BC. Without prestress, there was little radial compressive stress on the core stabilized soil in the early stage of loading. Only when the stabilized soil produced considerable lateral dilation deformation, could the radial compressive stress be produced passively. So the constraint pipe might hardly provide effective radial confining force to the core stabilized soil before it damaged. However, when bearing radial compressive prestress, the stabilized soil was in triaxial compressive stress state from the beginning of loading. As the existence of the radial pressure hindered the radial dilation of the stabilized soil, higher vertical pressure was needed to make the stabilized soil reach its ultimate tensile strain. Above analysis was supported by the facts in Figure 4 in which the linear stage of the CSSC lasted longer than that of the unconfined ones. Obviously, the greater the radial compressive prestress acted on the stabilized soil, the longer the linear stage lasted. Also the prestress improved the stress state of the core stabilized soil in the second stage of the load-displacement curves, which also improved the bearing performance and deformation characteristics of the CSSC.

\subsection{Influence of CS and Soil Density}

As shown in Figure 2(a), the BC increment of the CSSC was not significant as the thickness of the constraint pipe increased from $2.2 \mathrm{~mm}$ to $2.5 \mathrm{~mm}$, but it increased obviously when the thickness increased from $2.5 \mathrm{~mm}$ to $3.2 \mathrm{~mm}$. As shown in Figure 4, BC of the USSC of C13-2 and C13-3 were roughly same. When the thickness of the constraint pipe was $2.5 \mathrm{~mm}, \mathrm{BC}$ and deformation quantity of the confined cylinders didn't increase significantly, compared with that of the unconfined ones; while, as the thickness of constraint pipe increased to $3.2 \mathrm{~mm}, \mathrm{BC}$ and 
deformation quantity of the confined cylinders increased greatly.

The USSC was destroyed due to the vertical crack produced by the transverse tension stress as shown in Figure 3(a). C13-2 was destroyed due to the fracture of the confining pipe caused by transverse tension as shown in Figure 3(b), and failure mode of the core stabilized soil of C13-2 shown in Figure 3(c), was similar to that in Figure 3(a). C13-3 bulged outwards obviously before failure as shown in Figure 3(d). And the core stabilized soil of corrupted C13-3 only bulged without vertical crack as shown in Figure 3(e).

These results revealed the failure of all of the CSSC were due to the constraint pipe losing efficacy. Only the constraint pipe possessing sufficient CS, could the CSSC enhance effectively their $\mathrm{BC}$ and take favorable failure mode.

As shown in Figure 2(a), the bearing characteristics of samples $\mathrm{C} 13$ were respectively similar to those of samples C30 although density difference of the two core stabilized soil (shown in water content) was rather large. These results demonstrated that whether the CSSC made of plastic state soil or those made of plastic-flow state soil, which represented the range of soil used in engineering practice, all could improve $\mathrm{BC}$ effectively.

\subsection{Prospective Use of Confined Stabilized Soil}

The stabilized soil pile is a widely used foundation treatment method, which can make full use of the soft soil in situ and need not transport outward the abandoned soil as well as need carry much less pile materials into the site than other piles. But the low BC of the stabilized soil pile limited its application scope. Based on above results it was expected that the CSS technique might provide an effective foundation treatment method which could retain the advantages of the existing stabilized soil technique and also obtain rather high $\mathrm{BC}$.

Salt content of the saline soil around the Qarhan Salt Lake in Qinghai Province of China is even as high as 40 wt. \%. All kinds of pile foundation and foundation treatment methods containing Portland cement could not be used in the area for severe corrosion caused by the saline soil. The research of our team showed that strength of the stabilized saline soil stabilized with a special binder could increase to $11 \mathrm{MPa}$ and keep stable in the saline soil [5], combining which with the technique of the CSSP is expected to provide saline soil area with a high $\mathrm{BC}$ foundation treatment methods.

\section{Conclusion}

In the paper axial compression performance of the CSSC was investigated, following conclusions were gained. 1) The combination of the constraint pipe and the binder containing expansion component provided the stabilized soil with high strength. Made with same binder content, the CSSC could reach more than twice BC of the unconfined ones, also the CSSC showed rather larger plastic deformation. 2) The improvement of the CSS may be due to three effects: (1) with the constraint pipe, higher production of AFt could be adopted to increase the voidfilling rate so as to further improve the strength of the core stabilized soil; (2) hoop constraint provided radial compressive force on the core stabilized soil so as to enhance the $\mathrm{BC}$; (3) with the constraint pipe $\mathrm{AFt}$ expansion produced radial compressive prestress on the core stabilized soil. 3) The failure of CSSC was almost all due to the constraint pipe losing efficacy. Only the constraint pipe possessing sufficient CS could the CSSC enhance effectively their $\mathrm{BC}$ and take favorable failure mode.

\section{Acknowledgments}

The research is funded by the National Natural Science Foundation of China (No. 51578033).

\section{References}

1. Huang $\mathrm{X}$, Li Zhanguo, Ning Jianguo, and Xu Sheng. Principle and Method of Optimization Design for Soft Soil binder[J]. Journal of Wuhan University of Technology-Mater, 2009, 24(1): 154-160.

2. Li Z G, Ning $J$ G, and Huang $X$. Discussion on the Reasons of Different Curing Effects of Cement Expansive Composite Soft Soil[J]. China Civil Engineering Journal, 2011, 33(3): 420-426.

3. Heon-Soo Chung, Keun-Hyeok Yang, Young-Ho Lee, and Hee-Chang Eun. Stress-strain curve of laterally confined concrete[J]. Engineering Structures, 2002, 24(9): 1153-1163.

4. Ciupala M.A., Pilakoutas K., Mortazavi A.A., and Taranu N. RC Columns Lateral Prestressing with Composites[J]. 2th International Conference on Advanced Polymer Composites for Structural Applications in Construction, 2004.

5. Pan Yang, Xin Huang, Jin Pi, Jianwei Yang. Study on new cementitious materials used for pile and stabilized soil in super saline soil[J]. Japanese Geotechnical Society Special Publication, 2016, 2(62): 2111-2114 